PROCEEDINGS OF THE

AMERICAN MATHEMATICAL SOCIETY

Volume 131, Number 6, Pages 1759-1761

S 0002-9939(03)07003-5

Article electronically published on January 15, 2003

\title{
HYPERCYCLIC OPERATORS ON NON-LOCALLY CONVEX SPACES
}

\author{
JOCHEN WENGENROTH \\ (Communicated by Jonathan M. Borwein)
}

\begin{abstract}
We transfer a number of fundamental results about hypercyclic operators on locally convex spaces (due to Ansari, Bès, Bourdon, Costakis, Feldman, and Peris) to the non-locally convex situation. This answers a problem posed by A. Peris [Multi-hypercyclic operators are hypercyclic, Math. Z. 236 (2001), 779-786].
\end{abstract}

During the past years much research has been done about hypercyclic operators; the article [6] contains a rather complete survey of results until 1999. A (continuous linear) operator $T: X \rightarrow X$ on a topological vector space $X$ is called hypercyclic if it admits a vector $x \in X$ having dense orbit $\operatorname{Orb}(x)=\left\{x, T x, T^{2} x, \ldots\right\}$ ( $x$ is then called a hypercyclic vector). The following theorem collects some of the recent fundamental results:

Theorem. Let $X$ be a locally convex space and let $T: X \rightarrow X$ be an operator.

(1) Ansari 1]: If $T$ is hypercyclic, then so is $T^{n}$ for each $n \in \mathbb{N}$.

(2) Bourdon [3], Bès [2]: If T is hypercyclic there is a dense invariant subspace of (except for 0) hypercyclic vectors.

(3) Costakis [5], Peris [8]: If T is multi-hypercyclic (i.e. there are finitely many vectors such that the union of their orbits is dense), then $T$ is hypercyclic.

(4) Bourdon, Feldman [4]: Each orbit is either everywhere dense or nowhere dense.

A. Peris asked in [8] whether in (3) local convexity is really needed and we now show that it is indeed not:

All parts of the Theorem hold for topological Vector spaces.

The only place in the proof of the Theorem where local convexity plays a role is the following lemma which, for hypercyclic operators, is due to P. Bourdon [3] (the complex case) and J. Bès [2] (the real case). Our proof for the non-locally convex case is quite similar to their arguments.

Received by the editors November 23, 2001.

2000 Mathematics Subject Classification. Primary 47A16, 46A16.

Key words and phrases. Hypercyclic operators, supercyclic operators, multi-hypercyclic operators.

The author is indebted to Alfredo Peris for several very helpful remarks on a former version of this note. 
Lemma. Let $T$ be a continuous linear operator on a topological vector space admitting a vector with somewhere dense orbit. Then for each non-zero polynomial $p$ the operator $p(T)$ has dense range.

Of course, the coefficients of the polynomial are assumed to be real if $X$ is a real topological vector space.

Proof. We first consider a complex topological vector space $X$. Since the complex polynomial factorizes and the composition of dense range operators has dense range we may assume $p(z)=z-\lambda$ for some $\lambda \in \mathbb{C}$.

We assume $L=\overline{(T-\lambda \mathrm{id})(X)} \neq X$ and consider the quotient map $q: X \rightarrow X / L$ which clearly vanishes on $L$ and thus satisfies $q \circ(T-\lambda$ id $)=0$. Inductively this yields $q \circ T^{n}=\lambda^{n} q$ for all $n \in \mathbb{N}$ and therefore

$$
q(\operatorname{Orb}(x))=\left\{\lambda^{n} q(x): n \in \mathbb{N}\right\}=: M
$$

where $x$ is a vector whose orbit is somewhere dense. Since $q$ is a quotient map, $q(\operatorname{Orb}(x))$ is somewhere dense, too. On the other hand, $M$ is contained in a onedimensional subspace of the separated (since $L$ is closed) topological vector space $X / L$, hence $M$ is nowhere dense if the dimension of $X / L$ is at least two. Otherwise $X / L$ is isomorphic to $\mathbb{C}$ and then (depending on $|\lambda|$ ) $M$ either consists of a null sequence, is contained in some circle, or is closed, and in any case nowhere dense, a contradiction.

Now let $X$ be a real topological vector space. If there is a polynomial $p$ such that $p(T)$ does not have dense range we could use similar arguments as in [2] to produce a finite-dimensional factor of the dynamical system $(X, T)$ with a somewhere dense orbit - indeed, by factorization it is enough to consider $p(t)=t^{2}-a t-b$ and then we would obtain that the linear map given by $\left(\begin{array}{ll}a & 1 \\ b & 0\end{array}\right)$ on $\mathbb{R}^{2}$ has a somewhere dense orbit - and elementary arguments show that this is impossible.

However, there is a simpler proof which was generously provided by A. Peris. Let $\widetilde{X}=X+i X$ and $\widetilde{T}(x+i y)=T(x)+i T(y)$ be the complexifications. Since $p(T)$ has dense range if and only if $p(\widetilde{T})=\widetilde{p(T)}$ has dense range, it is again enough to show that $\widetilde{T}-\lambda$ id has dense range for each $\lambda \in \mathbb{C}$. Assuming the contrary, we define as before $L$ as the closure of $(\widetilde{T}-\lambda$ id $)(\widetilde{X})$ and denote the quotient map $\widetilde{X} \rightarrow \widetilde{X} / L$ by $q$. If $\operatorname{Orb}(x)$ is somewhere dense in $X$, then $A=\operatorname{Orb}(x)+i \operatorname{Orb}(x)$ is somewhere dense in $\tilde{X}$. On the other hand, for $z=T^{n}(x)+i T^{m}(x) \in A$ we have $q(z)=\left(\lambda^{n}+i \lambda^{m}\right) q(x)$, hence the somewhere dense set $q(A)$ is contained in a onedimensional subspace which implies $\widetilde{X} / L \cong \mathbb{C}$ (or, in other words, that $q \in \widetilde{X}^{\prime}$ is an eigenvector of $\left.\widetilde{T}^{*}\right)$. Now, we can argue as in [8]: $Q(y)=|q(y)|$ defines a continuous and open map $X \rightarrow[0, \infty)$, hence $Q(\operatorname{Orb}(x))$ is somewhere dense contradicting

$$
Q\left(T^{n}(x)\right)=\left|q\left(T^{n}(x)\right)\right|=\left|q\left(\widetilde{T}^{n}(x)\right)\right|=\left|\lambda^{n} q(x)\right|=|\lambda|^{n}|q(x)| .
$$

The results about hypercyclicity stated in the theorem above have counterparts for supercyclic operators which, by definition, have an orbit whose scalar multiples are dense, i.e. there is $x \in X$ such that $\operatorname{Orb}(\langle x\rangle)=\left\{\alpha T^{n}(x): n \in \mathbb{N}, \alpha \in \mathbb{K}\right\}$ is dense $(\langle x\rangle$ denotes the linear span of $\{x\})$. For locally convex spaces, Peris $[8]$ proved that (in the obvious sense) multi-supercyclic operators are supercyclic and Bourdon and N. Feldman [4] even showed that $\operatorname{Orb}(\langle x\rangle)$ is either everywhere dense 
or nowhere dense for each vector individually. As for the hypercyclic case, local convexity was only used in the proof of the locally convex version of:

Lemma. Let $T$ be an operator on a topological vector space $X$ admitting a vector $x$ such that $\operatorname{Orb}(\langle x\rangle)$ is somewhere dense. Then there exists $\lambda \in \mathbb{C}$ such that $p(T)$ has dense range for each polynomial $p$ with $p(\lambda) \neq 0$.

Proof. Let us show the real case; the complex one is similar but simpler. If $p$ is a polynomial with $p(T)$ having non-dense range, there is a root $\lambda_{1} \in \mathbb{C}$ of $p$ such that $\widetilde{T}-\lambda_{1}$ id does not have dense range (where as before, $\widetilde{X}$ and $\widetilde{T}$ denote complexifications) and if the lemma were false we could find $\lambda_{2} \notin\left\{\lambda_{1}, \overline{\lambda_{1}}\right\}$ such that $\widetilde{T}-\lambda_{2}$ id has non-dense range, too. Again, we denote by $L_{j}$ the closures of $\left(\widetilde{T}-\lambda_{j}\right.$ id $)(\widetilde{X})$ and the corresponding quotient maps by $q_{j}$.

Since $\operatorname{Orb}(\langle x\rangle)+i \operatorname{Orb}(\langle x\rangle)$ is somewhere dense in $\widetilde{X}$, we again obtain $\widetilde{X} / L_{j} \cong \mathbb{C}$, i.e. $q_{j}$ is an eigenvector of $\widetilde{T}^{*}$ with respect to $\lambda_{j}$. If $q_{j}=\varphi_{j}+i \psi_{j}$ with real continuous linear functionals $\varphi_{j}$ and $\psi_{j}$ we obtain that either $\varphi_{1}$ or $\psi_{1}$ is linear independent of $\left\{\varphi_{2}, \psi_{2}\right\}$, since otherwise we could find $a, b \in \mathbb{C}$ such that $q_{2}=a q_{1}+b \overline{q_{1}}$ where $\overline{q_{1}}=\varphi_{1}-i \psi_{1}$ is an eigenvector with respect to $\overline{\lambda_{1}}$, contradicting the fact that eigenvectors with respect to different eigenvalues are linearly independent. Hence there is $y \in X$ such that $q_{1}(y) \neq 0$ and $q_{2}(y)=0$.

We fix a non-zero $\alpha \in \mathbb{R}$ and $n \in \mathbb{N}$ such that $u=\alpha T^{n}(x) \in A$ where $A$ is the interior of $\overline{\operatorname{Orb}(\langle x\rangle)}$. Since $A-u$ is a 0 -neighbourhood in $X$ there is $\varepsilon>0$ such that for $0 \leq \delta \leq \varepsilon$ we have $u+\delta y \in A \subseteq \overline{\operatorname{Orb}(\langle x\rangle)}$. For fixed $\delta$ with $q_{1}(u+\delta y) \neq 0$ we can thus choose sequences $\left(\beta_{l}\right)_{l \in \mathbb{N}}$ in $\mathbb{R}$ and $\left(k_{l}\right)_{l \in \mathbb{N}}$ in $\mathbb{N}$ such that $\beta_{l} T^{k_{l}}(x) \rightarrow u+\delta y$. From $\lambda_{1} \neq 0$ (as $\widetilde{T}$ has dense range) and $q_{1}(x) \neq 0\left(\operatorname{as} q_{1}(\operatorname{Orb}(\langle x\rangle)+i \operatorname{Orb}(\langle x\rangle))\right.$ is somewhere dense) we obtain for $l$ large enough

$$
\left|\frac{\lambda_{2}}{\lambda_{1}}\right|^{k_{l}}\left|\frac{q_{2}(x)}{q_{1}(x)}\right|=\left|\frac{q_{2}\left(\beta_{l} T^{k_{l}}(x)\right)}{q_{1}\left(\beta_{l} T^{k_{l}}(x)\right)}\right| \longrightarrow\left|\frac{q_{2}(u+\delta y)}{q_{1}(u+\delta y)}\right|=\left|\frac{q_{2}(u)}{q_{1}(u)+\delta q_{1}(y)}\right| .
$$

Since $q_{2}(u)=\alpha \lambda_{2}^{n} q_{2}(x) \neq 0$ this implies that $\left|q_{1}(u)+\delta q_{1}(y)\right|$ is independent of $\delta$ which contradicts $q_{1}(y) \neq 0$.

\section{REFERENCES}

[1] S.I. Ansari, Hypercyclic and cyclic vectors, J. Funct. Anal. 128 (1995), 374-383. MR 96h:47002

[2] J.P. Bès, Invariant manifolds of hypercyclic vectors for the real scalar case, Proc. Amer. Math. Soc. 127 (1999), 1801-1804. MR 99i:47002

[3] P. Bourdon, Invariant manifolds of hypercyclic vectors, Proc. Amer. Math. Soc. 118 (1993), 845-847. MR 93i:47002

[4] P.S. Bourdon and N.S. Feldman, Somewhere dense orbits are everywhere dense, preprint, Washington and Lee University, 2001.

[5] G. Costakis, On a conjecture of D. Herrero concerning hypercyclic operators, C. R. Acad. Sci. Paris Ser. I Math. 130 (2000), 179-182. MR 2001a:47012

[6] K.G. Große-Erdmann, Universal families and hypercyclic operators, Bull. Amer. Math. Soc. 36 (1999), 345-381. MR 2000c: 47001

[7] D.A. Herrero, Hypercyclic operators and chaos, J. Operator Theory 28 (1992), 93-103. MR 95g:47031

[8] A. Peris, Multi-hypercyclic operators are hypercyclic, Math. Z. 236 (2001), 779-786. MR 2002a:47008

FB IV - Mathematik, Universität Trier, D - 54286 Trier, Germany

E-mail address: wengen@uni-trier.de 